\title{
HEALTH-RELATED QUALITY OF LIFE OF EX-PRISONERS OF WAR AFFECTED BY POSTTRAUMATIC STRESS DISORDER 25 YEARS AFTER CAPTIVITY
}

\author{
Melita Jukić $^{1,2}$, Pavo Filaković ${ }^{3}$, Ivan Požgain ${ }^{2,4}$ \& Trpimir Glavina ${ }^{5}$ \\ ${ }^{I}$ County General Hospital Vukovar and Croatian Veterans' Hospital, Department of Psychiatry, Vukovar, Croatia \\ ${ }^{2}$ Josip Juraj Strossmayer University of Osijek, Faculty of Medicine, Osijek, Croatia \\ ${ }^{3}$ Josip Juraj Strossmayer University of Osijek, Faculty of Dental Medicine and Health, Osijek, Croatia \\ ${ }^{4}$ University Hospital Center Osijek, Psychiatric Clinic, Osijek, Croatia \\ ${ }^{5}$ Department of Psychiatry, University Hospital Centre Split, Split, Croatia
}

received: 22.11.2018;

revised: 15.2.2019;

accepted: 15.3 .2019

\section{SUMMARY}

Background: War captivity is one of the most difficult human experiences and can cause long-lasting effects on mental and physical health. Posttraumatic Stress Disorder (PTSD), as one of the frequent consequences of war trauma, is often associated with the psychiatric and/or somatic comorbidity. Therefore, PTSD results in impaired Health-Related Quality of Life (HRQoL). This study aimed to investigate the HRQoL in the Croatian Homeland War ex-POWs affected by PTSD, regarding the intensity of PTSD symptoms, sociodemographic characteristics and somatic comorbidity, and to identify predictors of poor HRQLL.

Subjects and methods: The study sample consisted of two groups (45 participants each) based on whether they were POWs or not (control group). All study participants were diagnosed with PTSD according to the ICD-10 criteria and had combat experience as active participants in defence of the Republic of Croatia during the Homeland War. The subjects were evaluated using the sociodemographic questionnaire, PTSD self-report checklist (PCL-5) and Short Form (SF-36) Health Survey questionnaire. The data on participants' physical diseases were collected from medical anamnesis and medical records in the last five years.

Results: In relation to ex-POWs, the control group had significantly smaller number of retirees, more unemployed persons, smaller number of married subjects, and higher number of divorced persons. Low socioeconomic status and intensity of PTSD symptoms has been confirmed as a significant predictor of impaired HRQoL in both subject groups. The most commonly PTSD associated physical diseases were musculosceletal, cardiovascular, and gastrointestinal diseases. Endocrine and metabolic diseases were more frequent in the ex-POW' group.

Conclusions: PTSD was associated with the HRQoL, whether the veterans were ex-POWs or not. The hypothesis that exposure of ex-POWs to the trauma of captivity experience impaired HRQoL to a greater extent, compared to the non-detained veterans, was not confirmed. Low socioeconomic status has proved to be the most significant predictor of poorer HRQLL.

Key words: Posttraumatic Stress Disorder - Health-Related Quality of Life - Ex-prisoners of War - somatic comorbidity

$* * * * *$

\section{INTRODUCTION}

According to estimates by the Croatian Government, during the Homeland War (1990-1996) at least one million people have been exposed to the war stress (Ministry of Croatian Veterans 1999). People were traumatized in different ways: as active participants in defence of the sovereignty of the Republic of Croatia, as civilians exposed to combat operations, or as refugees with a long-term exile from their homes. Over 7,600 persons from the Republic of Croatia (civilians and defenders) were detained in a prisoner of war (POW) camps (Ministry of Croatian Veterans 2014). According to the Croatian Association of Prisoners in Serbian Concentration Camps, the number is as high as about 30,000 people. The exact number of defenders among them is unknown. Captivity is one of the most difficult human experiences, especially for people exposed to the combat stress. Frequently, imprisonment represents a repetitive and prolonged trauma, induced intentionally and traumatization often takes place at the interpersonal level (Herman 1992, Başoğlu 2009). Research on the consequences of captivity indicates a high risk of developing various effects on the health, especially the mental health of the individual, and one of the most common disorders mentioned as a possible consequence of trauma is Posttraumatic Stress Disorder (PTSD) (Solomon et al. 2012).

PTSD occurs after exposure to extreme stressors, life-threatening situations or witnessing the life-threatening situations. According to the International Classification of Diseases (ICD-10), PTSD belongs to a group of anxiety disorders and according to the Diagnostic and Statistical Manual of Mental Disorders (DSM-5), to a group of stress-related disorders (World Health Organization 1992, American Psychiatric Association 2013). To establish a PTSD diagnosis, the requirement of exposure to a traumatic event must be satisfied, and according to the ICD-10, the clinical picture of PTSD is characterized by symptoms classified in three groups: symptoms of re-experiencing trauma, avoidance of trauma reminders and hyperarousal behaviour. In DSM-5, 
symptoms are divided into four groups and, besides the above-mentioned symptoms, a group of symptoms includes cognitive and mood disorders.

The prevalence of PTSD depends on the study group and, according to the previous studies, the prevalence in the general population is $1-14 \%$ (American Psychiatric Association 1994). More prevalence refers to subpopulations exposed to certain types of stressors, e.g., refugees or other stressors related to war events. Some studies relating to POWs from the Gulf War and World War II indicate a high prevalence of PTSD in this population, up to over $80 \%$ (Al-Turkait \& Ohaeri 2008, Rintamaki et al. 2009). Often, PTSD can last for decades after exposure to traumatic events, as confirmed by veteran populations from various wars, whether it is caused by life-threatening situations during combat, captivity, or witnessing to traumatic events (Weiss et al. 2003). A large study, conducted in the period from 1986 to 1998 at the 3016 American veterans who participated in combat operations in Vietnam, describes that $15 \%$ of veterans had PTSD, and another $11 \%$ had partial PTSD at the time of testing (Kulka et al. 1990). A study conducted on Israeli veterans describes the persistence of PTSD in $23.2 \%$ of detainees 30 years after the captivity, unlike a significantly lower percentage of $4.3 \%$ for non-detainees veterans of the Yom- Kippur War (Neria et al. 1998). PTSD is a disorder with high comorbidity, both in psychiatric and somatic disorders. Many studies deal with health and comorbidity disorder in the veteran population, including ex-POWs. Some point to more frequent somatic comorbidity among ex-POWs compared to veterans who were not detainees, even many years after the war. Studies on veterans from Vietnam War and World War II describe the high incidence of somatic disorders among ex-POWs (Nice et al. 1996, Creasy et al. 1999), considerably impaired health several decades after trauma (Page \& Brass 1991), and also indicate high mortality (Solomon et al. 2013).

The World Health Organization (1997) defines the Quality of Life (QoL) as an individual's perception of its position in the specific health, cultural, social, and environmental context. War veterans, especially those with combat PTSD, often have a reduced QoL (Schnurr et al. 2009). Also, Health-Related Quality of Life (HRQoL), can be defined as a particular domain of the QoL concept specifically related to the health, referring patient's functioning, the well-being and perception of general health in the physical, mental and social domains (Apolone \& Mosconi 1998). Measurement of QoL can be very individual because of the unique experiences, expectations, and perceptions of each person. Therefore, QoL measurement should include reference to many complex areas (Ahmadi et al. 2013, Swan et al. 2009). Numerous studies describe diminished functioning and strong association of PTSD and decreased HRQoL (Buckley et al. 2004, Mittal et al. 2006). Richardson et al. (2008) found significant deterioration of mental and physical health in veterans suffering from PTSD. Many studies suggest that PTSD treatment leads to improved QoL (Rapaport et al. 2002, Schnurr et al. 2006).

Following the previous studies of captivity trauma, long-term consequences for the ex-POWs' health can be expected. This study aimed to investigate the HRQoL in the Croatian Homeland War ex-POWs affected by PTSD considering the intensity of PTSD symptoms, sociodemographic characteristics, and somatic comorbidity. Considering the exposure of exPOWs to the trauma of combat and captivity experience, we have assumed significantly impaired HRQoL in the ex-POWs group compared to the group of veterans not detained.

\section{SUBJECTS AND METHODS}

\section{Subjects}

The study sample consisted of 90 subjects, Croatian war veterans who were treated at the Department of Psychiatry of the County General Hospital Vukovar and Croatian Veterans' Hospital during 2017. All study participants were diagnosed with PTSD according to the ICD-10 criteria and had combat experience as active participants in defence of the Republic of Croatia during the Homeland War. They were divided into two groups (45 participants each) based on whether they were POWs or not (control group). Since there were many more who were not POWs, this control group was chosen randomly. The study was approved by the Ethics Committee of the County General Hospital Vukovar and Croatian Veterans' Hospital, and by the Ethics Committee of the Faculty of Medicine in Osijek. All subjects gave informed consent for participation in the research and patient anonymity has been preserved during all stages of investigation.

\section{Methods}

A clinical interview was conducted with all participants, and data on physical diseases were collected. The subjects were evaluated using the sociodemographic questionnaire, PTSD self-report checklist (PCL-5) and Short Form (SF-36) Health Survey questionnaire. The sociodemographic questionnaire was designed by authors and included data on age, employment status, education, marital and socioeconomic status.

The sociodemographic data were coded as follows:

- Years of age $(1 \leq 45 \mathrm{y} ; 2=45-54 \mathrm{y} ; 3=55-64 \mathrm{y}$; $4 \geq 64 y$ );

- Employment status (1 = Unemployed; 2 = Employed; 3 = Retired);

- Education ( 1 = Elementary school; 2 = High school; $3=\mathrm{BSc} ; 4$ = MSc);

- Marital status ( 1 = Widower; 2 = Divorced; 3 = Unmarried; 4 = Married),

- Socioeconomic status ( 1 = Bad; 2 = Moderate; 3 = Good). 
The PCL-5 checklist containing 20 items corresponding to the DSM-5 symptom criteria for PTSD was used (American Psychiatric Association 2013, Weathers et al. 2014). The items are related to specific symptoms in the past month. Items were rated on a 5-point scale (0-"Not at all", 1-"A little bit", 2-"Moderately", 3-"Quite a bit", and 4-"Extremely") and four subscales (clusters) were formed: B-Intrusion (items 1-5), CAvoidance (items 6-7), D-Negative alterations in cognitions and mood (items 8-14), and E-Alterations in arousal and reactivity (items 15-20) (American Psychiatric Association 2013, Wortmann et al. 2016). Cluster scores were calculated as an average score of corresponding items. A total PCL-5 score was obtained by summing the scores for each item and ranged from 0 to 80 , where higher scores indicate more severe symptoms.

The Short Form (SF-36) Health Survey questionnaire contains 36 items (questions), 35 of which are distributed as a measure of 8 concepts (dimensions) of health: Physical functioning (PF) - 10 items, Physical role limitations (RP) -4 items, Bodily pain (BP) -2 items, General health $(\mathrm{GH})-5$ items, Vitality (VT) -4 items, Social functioning (SF) - 2 items, Emotional role limitations (RE) -3 items, and Mental health $(\mathrm{MH})-5$ items (Ware et al. 1994, Ware \& Kosinski 2001). The original scores of SF-36 questionnaire were adjusted by summing and rescaling the factor-weighted scores to fit eight dimensions of health with a range from 0 to 100 , where a score of 100 indicates the best health condition. These eight dimensions of health have also been distributed and summarized into two scales: a Physical component score (PCS) and Mental component score (MCS) (Ware et al. 1993, Ware et al. 2001). The Total SF-36 score was obtained by averaging scores for all eight dimensions.

The data on participants' physical diseases were collected from medical anamnesis and medical records in the last five years. The data was divided into Cardiovascular (CVD), Gastrointestinal (GID), Neurological (ND), Endocrine and metabolic (EMD), Musculosceletal (MSD), Malignant (MD), Dermatological (DD), and Urological (UD) diseases groups.

\section{Statistical Analyses}

To describe the study sample, frequencies were calculated for sociodemographic and physical diseases data, and mean and standard deviation (SD) were calculated for data on PTSD self-report checklist (PCL-5) and Short Form (SF-36) Health Survey questionnaire. Chi-Square $\left(\chi^{2}\right)$ and Mann-Whitney U tests were used to examine the potential differences in those variables between the ex-POWs and the control group.

A generalized regression model analysis was conducted to identify factors affecting patients' QoL. First, univariate analysis was performed to determine the difference between subject groups, and second, the regression model was adjusted for age, employment status, education level, marital and socioeconomic status. In addition, logistic regression was performed to determine the odds ratio (OR) for physical disease morbidity in the ex-POWs and the control group. A specific physical disease was recorded into variables with two levels $(1=$ diagnosed; $0=$ not diagnosed $)$. The adjusted odds ratios were also calculated after including the possible confounding sociodemographic factors in the logistic regression model.

Associations between the data on participants' physical diseases and the PCL-5 and SF-36 scores were evaluated with the Spearman's rank correlations. Dermatological and urological diseases have been removed from the regression and correlation analyses because their incidence among subject groups was not significant $(<5 \%)$. Statistical analysis was carried out with the Statistica ver. 13.1 software (Dell Inc., Tulsa, OK, USA).

\section{RESULTS}

Data on the sociodemographic characteristics of subjects are presented in Table 1 . There were no statistically significant differences $(p<0.05)$ in age and the level of education of the ex-POWs and the control group. Most subjects were in age between 45 and 64 years and had a high school level of education. A significant difference was found in the employment $\left(\chi^{2}=11.11 ; \quad \mathrm{p}=0.004\right)$ and marital status $\left(\chi^{2}=10.98\right.$; $\mathrm{p}=0.012$ ), and in the self-assessed socioeconomic status $\left(\chi^{2}=9.78 ; p=0.008\right)$. The control group had a significantly smaller number of retirees in relation to exPOWs, 62.2 and $86.7 \%$, respectively. There were significantly more unemployed persons in the control group ( $26.7 \%$ vs. $2.2 \%$ in ex-POWs), while the number of employed subjects was the same in both groups. There were no unmarried and widowers in the ex-POWs group, and the number of married subjects was significantly higher in relation to the control group $(91.1 \%$ vs. $64.4 \%)$. The number of divorced persons was significantly higher in the control group $(20.0 \%$ vs. $8.9 \%$ in ex-POWs). Low socioeconomic status was significantly more prevalent in the control group (44.4\% vs. $15.6 \%$ in ex-POWs).

Table 2 shows comparison of the PCL-5 cluster scores in ex-POWs and the control group. According to Mann-Whitney $U$ test, there were a significant differences $(p<0.05)$ in the three PCL-5 clusters: B-Intrusion $(\mathrm{Z}=-2.557 ; \mathrm{p}=0.011)$, $\mathrm{D}-\mathrm{Cognition}$ and $\operatorname{mood}(\mathrm{Z}=-2.028$; $\mathrm{p}=0.043)$, and $\mathrm{E}$-Arousal and reactivity $(\mathrm{Z}=-2.241$; $\mathrm{p}=0.025$ ). All these cluster symptoms were more pronounced in the control group. The total PCL-5 score was significantly lower $(\mathrm{Z}=-2.038 ; \mathrm{p}=0.042)$ in the exPOWs compared to the control group (33.7 vs. 42.0), indicating the lower intensity of PTSD symptoms in the ex-POWs group.

The data on participants' physical diseases are presented in Table 3. The most commonly PTSD associated physical diseases were musculosceletal, cardiovascular, and gastrointestinal diseases, both in the group of ex-POWs and in the control group. Endocrine and 
Table 1. Differences in the sociodemographic data between ex-POWs and the control group

\begin{tabular}{|c|c|c|c|c|c|c|}
\hline & \multicolumn{2}{|c|}{ ex-POWs } & \multicolumn{2}{|c|}{ Control } & \multirow[b]{2}{*}{$\chi^{2}$} & \multirow[b]{2}{*}{$\mathrm{p}$} \\
\hline & $\mathrm{n}$ & $\%$ & $\mathrm{n}$ & $\%$ & & \\
\hline Age & & & & & 3.815 & 0.282 \\
\hline$<45$ & 1 & 2.2 & 5 & 11.1 & & \\
\hline $45-54$ & 21 & 46.7 & 22 & 48.9 & & \\
\hline $55-64$ & 17 & 37.8 & 15 & 33.3 & & \\
\hline$>64$ & 6 & 13.3 & 3 & 6.7 & & \\
\hline Employment status & & & & & 11.114 & $0.004^{*}$ \\
\hline Unemployed & 1 & 2.2 & 12 & 26.7 & & \\
\hline Employed & 5 & 11.1 & 5 & 11.1 & & \\
\hline Retired & 39 & 86.7 & 28 & 62.2 & & \\
\hline Education & & & & & 2.406 & 0.493 \\
\hline Elementary school & 12 & 26.7 & 10 & 22.2 & & \\
\hline High school & 27 & 60.0 & 32 & 71.1 & & \\
\hline $\mathrm{BSc}$ & 2 & 4.4 & 2 & 4.4 & & \\
\hline $\mathrm{MSc}$ & 4 & 8.9 & 1 & 2.2 & & \\
\hline Marital status & & & & & 10.980 & $0.012 *$ \\
\hline Widower & 0 & 0.0 & 1 & 2.2 & & \\
\hline Divorced & 4 & 8.9 & 9 & 20.0 & & \\
\hline Unmarried & 0 & 0.0 & 6 & 13.3 & & \\
\hline Married & 41 & 91.1 & 29 & 64.4 & & \\
\hline Socioeconomic status & & & & & 9.783 & $0.008 *$ \\
\hline $\mathrm{Bad}$ & 7 & 15.6 & 20 & 44.4 & & \\
\hline Moderate & 34 & 75.6 & 24 & 53.3 & & \\
\hline Good & 4 & 8.9 & 1 & 2.2 & & \\
\hline
\end{tabular}

Table 2. Differences in the PCL-5 cluster scores between ex-POWs and the control group

\begin{tabular}{|c|c|c|c|c|c|c|}
\hline \multirow{2}{*}{ PCL-5 Clusters } & \multicolumn{2}{|c|}{ ex-POWs } & \multicolumn{2}{|c|}{ Control } & \multirow[b]{2}{*}{ Z } & \multirow[b]{2}{*}{$\mathrm{p}$} \\
\hline & Mean & SD & Mean & SD & & \\
\hline B-Intrusion & 1.8 & 0.9 & 2.3 & 0.9 & -2.557 & $0.011 *$ \\
\hline C-Avoidance & 2.0 & 1.4 & 2.0 & 1.0 & -0.016 & 0.987 \\
\hline D-Cognition and mood & 1.6 & 1.1 & 2.0 & 0.9 & -2.028 & $0.043 *$ \\
\hline E-Arousal and reactivity & 1.6 & 1.0 & 2.1 & 0.9 & -2.241 & $0.025 *$ \\
\hline Total PCL-5 & 33.7 & 18.5 & 42.0 & 16.6 & -2.038 & $0.042 *$ \\
\hline
\end{tabular}

$* \mathrm{p}<0.05$

Table 3. Comparison of the physical diseases morbidity in ex-POWs and the control group

\begin{tabular}{|c|c|c|c|c|c|c|}
\hline \multirow{2}{*}{ Disease } & \multicolumn{2}{|c|}{ ex-POWs } & \multicolumn{2}{|c|}{ Control } & \multirow[b]{2}{*}{$\chi^{2}$} & \multirow[b]{2}{*}{$\mathrm{p}$} \\
\hline & $\mathrm{n}$ & $\%$ & $\mathrm{n}$ & $\%$ & & \\
\hline Cardiovascular & 23 & 51.1 & 22 & 48.9 & 0.044 & 0.833 \\
\hline Gastrointestinal & 18 & 40.0 & 15 & 33.3 & 0.431 & 0.512 \\
\hline Neurological & 6 & 13.3 & 12 & 26.7 & 2.500 & 0.114 \\
\hline Endocrine and metabolic & 14 & 31.1 & 6 & 13.3 & 4.114 & $0.043^{*}$ \\
\hline Musculosceletal & 28 & 62.2 & 25 & 55.6 & 0.413 & 0.520 \\
\hline Malignant & 5 & 11.1 & 3 & 6.7 & 0.549 & 0.459 \\
\hline Dermatological & 1 & 2.2 & 2 & 4.4 & 0.345 & 0.557 \\
\hline Urological & 2 & 4.4 & 1 & 2.2 & 0.345 & 0.557 \\
\hline
\end{tabular}

$* \mathrm{p}<0.05$

metabolic diseases were significantly more frequent $\left(\chi^{2}=4.114 ; \mathrm{p}=0.043\right)$ in the group of ex-POWs than in the control group (31.1\% vs. 13.3\%). Neurological diseases occurred more frequently in the control group, but no statistical significance was determined by the Chi-Square $\left(\chi^{2}\right)$ test $\left(\chi^{2}=2.500 ; p=0.114\right)$.
The results of the Short Form (SF-36) Health Survey questionnaire showed that the TSF36-Total SF-36 score in the group of ex-POWs was significantly higher than in the control group $(Z=2.227 ; p=0.026)$ according to Mann-Whitney U test (Table 4). The statistically significant difference has also been found in the MSC-Mental 
component score $(Z=2.570 ; p=0.01)$, which was also higher in the ex-POWs group. The total PSC-Physical component score did not show a significant difference between groups. The results of individual subscales scores showed differences in several dimensions of health; in the BP-Bodily pain dimension $(\mathrm{Z}=2.110 ; \mathrm{p}=0.035)$ with more severe pain in the control group, and in the three mental components of health: SF-Social functioning $(Z=2.108 ; \quad p=0.035)$, RE-Emotional roles limitation $(Z=2.161 ; p=0.031)$, and MH-Mental health $(Z=3.276$; $\mathrm{p}=0.001)$. In all these dimensions, the control group had a lower score, indicating poorer social functioning, more impaired functioning due to emotional roles limitation and worse mental health compared to the ex-POWs.

Table 5 shows standardized $\beta$ - coefficients from the regression analysis of the PCL-5 cluster scores. The results of unadjusted univariate regression confirmed that the control group was statistically significantly $(\mathrm{p}<0.05)$ more likely to develop more severe PTSD symptoms compared with ex-POWs group. It was evident from the obtained $\beta$ - coefficients for the TPCL5-Total PCL-5 score $(\beta=-0.233 ; p=0.027)$, and individual clusters: $B-$ Intrusion $(\beta=-0.270 ; p=0.010), D-N e g a t i v e$ alterations in cognitions and $\operatorname{mood}(\beta=-0.213 ; p=0.044)$, and E-Alterations in arousal and reactivity $(\beta=-0.242 ; p=0.021)$. However, when the adjusted linear regression analysis was applied, none of the group related $\beta$ - coefficients maintained their significance. The bad socioeconomic status appeared to be the most important predictor of the more intense PTSD symptoms in the clusters $D(\beta=-0.309$; $\mathrm{p}=0.010)$ and $\mathrm{E}(\beta=-0.281 ; \mathrm{p}=0.016)$, and the TPCL5Total PCL-5 score $(\beta=-0.277 ; p=0.020)$. Lower age of participants emerged as a significant predictor of the cluster E symptoms $(\beta=-0.217 ; p=0.035)$.

Table 4. Differences in the SF-36 health survey scores between ex-POWs and the control group

\begin{tabular}{|c|c|c|c|c|c|c|}
\hline \multirow{2}{*}{ SF-8 dimensions } & \multicolumn{2}{|c|}{ ex-POWs } & \multicolumn{2}{|c|}{ Control } & \multirow[b]{2}{*}{ Z } & \multirow[b]{2}{*}{$\mathrm{p}$} \\
\hline & Mean & $\mathrm{SD}$ & Mean & SD & & \\
\hline $\mathrm{PF}$ & 52.6 & 23.8 & 49.2 & 22.0 & 0.862 & 0.389 \\
\hline $\mathrm{RP}$ & 20.6 & 30.3 & 17.8 & 26.4 & 0.144 & 0.885 \\
\hline BP & 48.2 & 18.9 & 40.4 & 19.0 & 2.110 & $0.035^{*}$ \\
\hline $\mathrm{GH}$ & 39.6 & 21.2 & 31.6 & 13.6 & 1.647 & 0.100 \\
\hline VT & 37.2 & 18.9 & 30.7 & 18.6 & 1.791 & 0.073 \\
\hline SF & 47.2 & 22.3 & 36.7 & 21.6 & 2.108 & $0.035 *$ \\
\hline RE & 27.4 & 39.8 & 11.9 & 27.7 & 2.161 & $0.031 *$ \\
\hline $\mathrm{MH}$ & 47.9 & 16.7 & 35.2 & 16.9 & 3.276 & $0.001 *$ \\
\hline PCS & 40.2 & 16.5 & 34.7 & 13.8 & 1.635 & 0.102 \\
\hline MCS & 39.9 & 20.9 & 28.6 & 15.3 & 2.570 & $0.010 *$ \\
\hline TSF36 & 40.1 & 17.0 & 31.7 & 13.4 & 2.227 & $0.026 *$ \\
\hline
\end{tabular}

PF - Physical functioning; RP - Physical roles limitation; BP - Bodily pain; GH - General health; VT - Vitality; SF - Social functioning; RE - Emotional roles limitation; MH - Mental health; PCS - Physical component score; MCS - Mental component score; TSF36 - Total SF-36; $\quad * \mathrm{p}<0.05$

Table 5. Regression analysis and factors associated with the self-report checklist PCL-5 cluster scores

\begin{tabular}{|c|c|c|c|c|c|c|c|c|}
\hline & \multirow[b]{2}{*}{ Parameter } & \multirow{2}{*}{$\begin{array}{c}\text { Univariate } \\
\text { exPOWs/ Control }\end{array}$} & \multicolumn{6}{|c|}{ Adjusted } \\
\hline & & & exPOWs/ Control & AGE & $\mathrm{ES}$ & ED & MS & SEC \\
\hline \multirow[t]{3}{*}{$\mathrm{B}$} & $\beta$ & -0.270 & -0.194 & -0.097 & -0.072 & -0.100 & 0.076 & -0.153 \\
\hline & $95 \% \mathrm{CI}$ & $-0.47-0.07$ & $-0.42-0.03$ & $-0.3-0.11$ & $-0.31-0.17$ & $-0.32-0.11$ & $-0.14-0.29$ & $-0.39-0.08$ \\
\hline & $\mathrm{p}$ & $0.010 *$ & 0.093 & 0.357 & 0.547 & 0.356 & 0.485 & 0.198 \\
\hline \multirow[t]{3}{*}{$\mathrm{C}$} & $\beta$ & 0.000 & -0.008 & -0.003 & 0.049 & -0.038 & 0.186 & -0.174 \\
\hline & $95 \% \mathrm{CI}$ & $-0.21-0.21$ & $-0.25-0.23$ & $-0.22-0.22$ & $-0.2-0.3$ & $-0.26-0.19$ & $-0.04-0.41$ & $-0.42-0.07$ \\
\hline & $\mathrm{p}$ & 1.000 & 0.947 & 0.981 & 0.697 & 0.737 & 0.106 & 0.163 \\
\hline \multirow[t]{3}{*}{$\mathrm{D}$} & $\beta$ & -0.213 & -0.149 & -0.146 & 0.120 & -0.079 & 0.099 & -0.309 \\
\hline & $95 \% \mathrm{CI}$ & $-0.42-0.01$ & $-0.37-0.08$ & $-0.35-0.06$ & $-0.12-0.36$ & $-0.29-0.13$ & $-0.12-0.31$ & $-0.54-0.08$ \\
\hline & $\mathrm{p}$ & $0.044^{*}$ & 0.190 & 0.163 & 0.317 & 0.463 & 0.362 & $0.010^{*}$ \\
\hline \multirow[t]{3}{*}{$\mathrm{E}$} & $\beta$ & -0.242 & -0.205 & -0.217 & 0.149 & -0.052 & 0.168 & -0.281 \\
\hline & $95 \% \mathrm{CI}$ & $-0.45-0.04$ & $-0.43-0.01$ & $-0.42--0.02$ & $-0.08-0.38$ & $-0.26-0.16$ & $-0.04-0.38$ & $-0.51-0.05$ \\
\hline & $\mathrm{p}$ & $0.021 *$ & 0.067 & $0.035^{*}$ & 0.203 & 0.625 & 0.115 & $0.016^{*}$ \\
\hline \multirow[t]{3}{*}{ TPCL5 } & $\beta$ & -0.233 & -0.177 & -0.153 & 0.083 & -0.079 & 0.138 & -0.277 \\
\hline & $95 \% \mathrm{CI}$ & $-0.44-0.03$ & $-0.4-0.05$ & $-0.36-0.05$ & $-0.15-0.32$ & $-0.29-0.13$ & $-0.08-0.35$ & $-0.51-0.05$ \\
\hline & p & $0.027^{*}$ & 0.121 & 0.142 & 0.485 & 0.459 & 0.202 & $0.020 *$ \\
\hline
\end{tabular}

$\beta$ - standardized beta coefficient; CI - Confidence interval; $\mathrm{B}$ - Intrusion; $\mathrm{C}$ - Avoidance; $\mathrm{D}$ - Cognition and mood;

E - Arousal and reactivity; TPCL5 - Total PCL-5 score; ES - Employment status; ED - Education; MS - Marital status;

SEC - Socioeconomic status; $\quad * \mathrm{p}<0.05$ 
The odds ratios obtained from the logistic regression analysis showed that the only group of diseases with a statistically significant difference between subject groups was a group of endocrinal and metabolic diseases $(\mathrm{p}=0.040)$. There were 2.94 times more likely to develop these diseases in the ex-POWs group compared to the control group. After the adjustment for the possible confounding factors, the only significant predictor of physical diseases was the marital status as a predictor of cardiovascular diseases $(\beta=-0.362 ; p=0.009)$, indicating the better cardiovascular health condition of married participants (Table 6).

Table 6. Regression analysis and factors associated with the physical diseases morbidity in ex-POWs and the control group

\begin{tabular}{|c|c|c|c|c|c|c|c|c|}
\hline & \multirow[b]{2}{*}{ Parameter } & \multicolumn{2}{|l|}{ Univariate } & \multicolumn{4}{|c|}{ Adjusted } & \multirow[b]{2}{*}{ SEC } \\
\hline & & exPOWs/Control & exPOWs/Control & AGE & $\mathrm{ES}$ & ED & MS & \\
\hline \multirow[t]{3}{*}{ CVD } & $\beta(\mathrm{OR})$ & $0.025(1.09)$ & $0.108(1.48)$ & 0.194 & 0.099 & 0.154 & -0.362 & -0.208 \\
\hline & $95 \% \mathrm{CI}$ & $-0.2-0.25$ & $-0.16-0.38$ & $-0.06-0.45$ & $-0.19-0.39$ & $-0.11-0.42$ & $-0.66-0.07$ & $-0.5-0.08$ \\
\hline & $\mathrm{p}$ & 0.833 & 0.433 & 0.126 & 0.506 & 0.240 & $0.009^{*}$ & 0.156 \\
\hline \multirow[t]{3}{*}{ GID } & $\beta(\mathrm{OR})$ & $0.079(1.33)$ & $0.055(1.22)$ & 0.138 & 0.120 & 0.009 & -0.140 & 0.008 \\
\hline & $95 \% \mathrm{CI}$ & $-0.16-0.32$ & $-0.21-0.32$ & $-0.11-0.38$ & $-0.17-0.41$ & $-0.24-0.26$ & $-0.39-0.11$ & $-0.27-0.28$ \\
\hline & $\mathrm{p}$ & 0.511 & 0.686 & 0.271 & 0.416 & 0.943 & 0.281 & 0.957 \\
\hline \multirow[t]{3}{*}{ ND } & $\beta(\mathrm{OR})$ & $-0.237(0.42)$ & $-0.118(0.65)$ & 0.020 & -0.235 & 0.150 & -0.145 & -0.028 \\
\hline & $95 \% \mathrm{CI}$ & $-0.54-0.06$ & $-0.46-0.22$ & $-0.29-0.33$ & $-0.55-0.08$ & $-0.16-0.46$ & $-0.43-0.14$ & $-0.36-0.3$ \\
\hline & $\mathrm{p}$ & 0.111 & 0.239 & 0.113 & 0.546 & 0.705 & 0.647 & 0.650 \\
\hline \multirow[t]{3}{*}{ EMD } & $\beta(\mathrm{OR})$ & 0.297 (2.94) & $0.192(2.00)$ & 0.236 & 0.121 & -0.055 & 0.083 & 0.077 \\
\hline & $95 \% \mathrm{CI}$ & $0-0.59$ & $-0.13-0.52$ & $-0.06-0.53$ & $-0.28-0.52$ & $-0.34-0.23$ & $-0.28-0.45$ & $-0.26-0.41$ \\
\hline & $\mathrm{p}$ & $0.040 *$ & 0.497 & 0.898 & 0.139 & 0.357 & 0.319 & 0.868 \\
\hline \multirow[t]{3}{*}{ LMD } & $\beta(\mathrm{OR})$ & $0.076(1.32)$ & $0.080(1.34)$ & 0.094 & 0.003 & 0.006 & 0.041 & -0.098 \\
\hline & $95 \% \mathrm{CI}$ & $-0.16-0.31$ & $-0.18-0.34$ & $-0.15-0.34$ & $-0.27-0.28$ & $-0.24-0.26$ & $-0.2-0.29$ & $-0.37-0.17$ \\
\hline & $\mathrm{p}$ & 0.520 & 0.548 & 0.444 & 0.983 & 0.960 & 0.744 & 0.476 \\
\hline \multirow[t]{3}{*}{ MD } & $\beta(\mathrm{OR})$ & $0.154(1.75)$ & $0.092(1.40)$ & -0.213 & -0.025 & -0.435 & -0.099 & 0.398 \\
\hline & $95 \% \mathrm{CI}$ & $-0.26-0.57$ & $-0.4-0.59$ & $-0.7-0.27$ & $-0.56-0.5$ & $-1.02-0.15$ & $-0.53-0.33$ & $-0.09-0.89$ \\
\hline & $\mathrm{p}$ & 0.457 & 0.714 & 0.373 & 0.926 & 0.103 & 0.658 & 0.103 \\
\hline
\end{tabular}

$\beta$ - standardized beta coefficient; OR - Odds ratio; CI - Confidence interval; CVD - Cardiovascular diseases;

GID - Gastrointestinal diseases; ND - Neurological diseases; EMD - Endocrine and metabolic diseases;

MSD - Musculosceletal diseases; MD - Malignant diseases; TPCL5 - Total PCL-5 score; ES - Employment status;

ED - Education; MS - Marital status; SEC - Socioeconomic status; $\quad * \mathrm{p}<0.05$

Table 7. Spearman rank order correlations between SF-36 and PCL-5 scores and physical morbidity

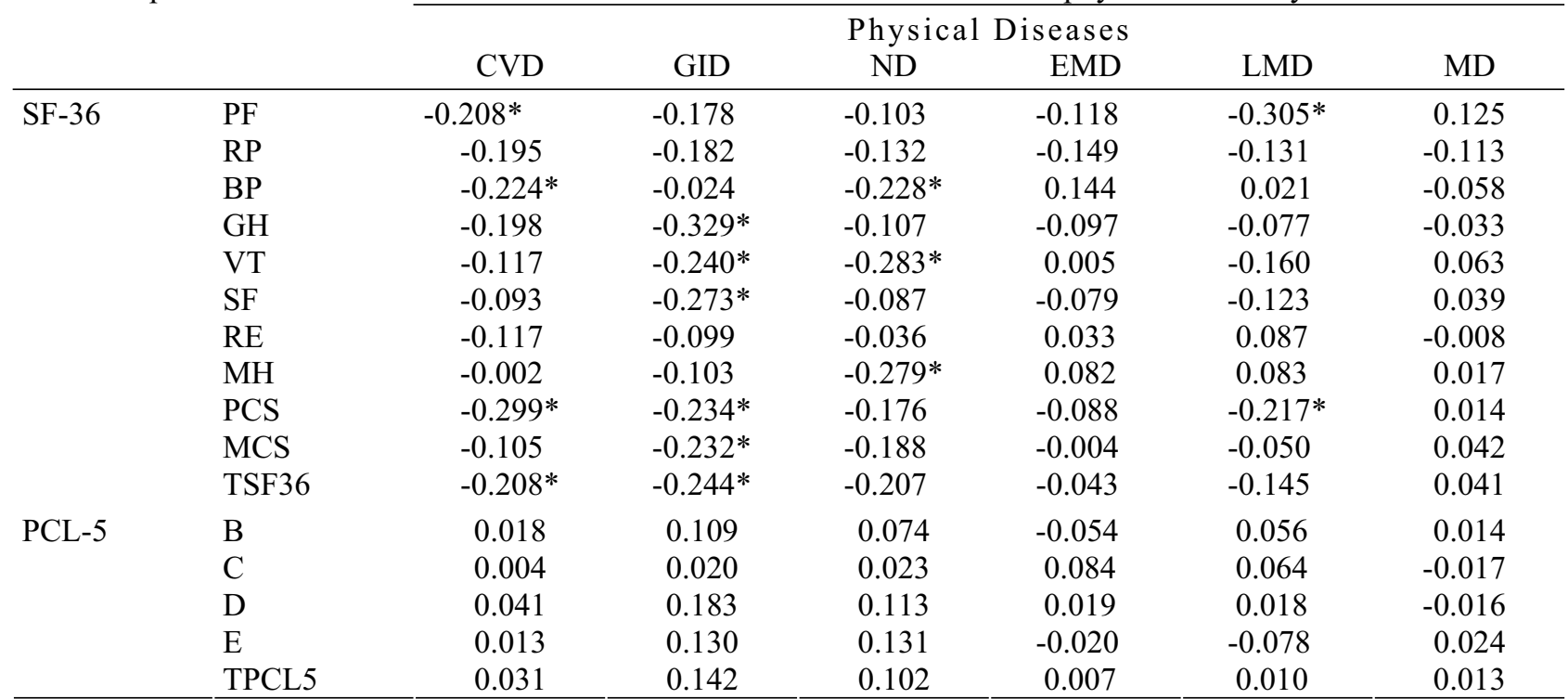

PF - Physical functioning; RP - Physical roles limitation; BP - Bodily pain; GH - General health; VT - Vitality;

SF - Social functioning; RE - Emotional roles limitation; MH - Mental health; PCS - Physical component score;

MCS - Mental component score; TSF36 - Total SF-36; B - Intrusion; C - Avoidance; D - Cognition and mood;

E - Arousal and reactivity; TPCL5 - Total PCL-5 score; CVD - Cardiovascular diseases; GID - Gastrointestinal diseases;

ND - Neurological diseases; EMD - Endocrine and metabolic diseases; MSD - Musculosceletal diseases; MD - Malignant diseases;

$* \mathrm{p}<0.05$ 
Spearman's rho $(\rho)$ correlation coefficients between SF-36 and PCL-5 scores and physical morbidity are presented in Table 7. There was a significant correlation $(p<0.05)$ between the cardiovascular diseases incidence and the total SF-36 score $(\rho=-0.208)$, primarily due to correlation with the PCS-Physical component score $(\rho=-0.299)$ with a significant influence of the PFPhysical functioning and BP-Bodily pain dimensions ( $\rho=-0.208$ and $\rho=-0.224$, respectively). The gastrointestinal diseases among subjects significantly correlated with the total SF-36 score $(\rho=0.244)$, PCS-Physical component score $(\rho=-0.234)$, and MSC-Mental component score $(\rho=-0.232)$, which is related with impaired GH-General health $(\rho=-0.329)$, VT-Vitality $(\rho=-0.240)$, and SF-Social functioning $(\rho=-0.273)$. Development of the neurological diseases significantly correlated with the BP-Bodily pain dimension $(\rho=-0.228)$, and bad perception of the VT-Vitality $(\rho=-0.283)$ and MH-Mental health $(\rho=-0.279)$. The prevalence of musculosceletal diseases correlated with the PCS-Physical component score $(\rho=0.217)$, mainly due to correlation with a deterioration of PF-Physical functioning $(\rho=-0.305)$. None of the PCL-5 cluster has correlate with any group of physical diseases.

The overall results of the regression analysis for the 8 -dimensional SP-36 health survey in Table 8 showed that ex-POWs assessed their health as better compared to the control group. The statistically significant differences $(p<0.05)$ have been found in the health dimensions of the GH-General health $(\beta=-0.221$; $\mathrm{p}=0.036)$, SF-Social functioning $(\beta=-0.237 ; \mathrm{p}=0.025)$, RE-Emotional roles limitation $(\beta=-0.224 ; p=0.034)$, and $\mathrm{MH}-\mathrm{Mental}$ health $(\beta=-0.358 ; \mathrm{p}=0.001)$. Consequently, scores for the MSC-Mental component score $(\beta=-0.299$;

Table 8. Regression analysis and factors associated with the 8-dimensional SP-36 health survey

\begin{tabular}{|c|c|c|c|c|c|c|c|c|}
\hline & \multirow[b]{2}{*}{ Parameter } & \multicolumn{2}{|l|}{ Univariate } & \multicolumn{4}{|c|}{ Adjusted } & \multirow[b]{2}{*}{ SEC } \\
\hline & & ex-POWs/ Control & ex-POWs/ Control & AGE & ES & ED & MS & \\
\hline \multirow[t]{3}{*}{$\overline{P F}$} & $\beta$ & 0.073 & 0.033 & -0.142 & 0.039 & -0.048 & -0.052 & 0.213 \\
\hline & $95 \% \mathrm{CI}$ & $-0.14-0.28$ & $-0.2-0.27$ & $-0.36-0.07$ & $-0.21-0.29$ & $-0.27-0.18$ & $-0.28-0.17$ & $-0.03-0.46$ \\
\hline & $\mathrm{p}$ & 0.492 & 0.784 & 0.196 & 0.756 & 0.672 & 0.648 & 0.086 \\
\hline \multirow[t]{3}{*}{$\mathrm{RP}$} & $\beta$ & 0.049 & 0.044 & -0.085 & -0.107 & 0.089 & -0.012 & 0.165 \\
\hline & $95 \% \mathrm{CI}$ & $-0.16-0.26$ & $-0.2-0.28$ & $-0.3-0.13$ & $-0.36-0.14$ & $-0.14-0.32$ & $-0.24-0.22$ & $-0.08-0.41$ \\
\hline & $\mathrm{p}$ & 0.644 & 0.717 & 0.444 & 0.401 & 0.438 & 0.920 & 0.188 \\
\hline \multirow[t]{3}{*}{$\mathrm{BP}$} & $\beta$ & 0.203 & 0.191 & 0.022 & -0.196 & 0.197 & 0.014 & 0.177 \\
\hline & $95 \% \mathrm{CI}$ & $0-0.41$ & $-0.04-0.42$ & $-0.19-0.23$ & $-0.44-0.04$ & $-0.02-0.41$ & $-0.21-0.23$ & $-0.06-0.41$ \\
\hline & $\mathrm{p}$ & 0.055 & 0.124 & 0.638 & 0.105 & 0.111 & 0.750 & 0.010 \\
\hline \multirow[t]{3}{*}{ GH } & $\beta$ & 0.221 & 0.174 & -0.048 & -0.193 & 0.171 & 0.034 & 0.307 \\
\hline & $95 \% \mathrm{CI}$ & $0.01-0.43$ & $-0.05-0.4$ & $-0.25-0.16$ & $-0.43-0.04$ & $-0.04-0.38$ & $-0.18-0.25$ & $0.08-0.54$ \\
\hline & $\mathrm{p}$ & $0.036^{*}$ & 0.101 & 0.837 & 0.109 & 0.076 & 0.901 & $0.010^{*}$ \\
\hline \multirow[t]{3}{*}{ VT } & $\beta$ & 0.174 & 0.122 & -0.008 & -0.230 & 0.088 & 0.048 & 0.343 \\
\hline & $95 \% \mathrm{CI}$ & $-0.03-0.38$ & $-0.1-0.35$ & $-0.21-0.2$ & $-0.47-0.01$ & $-0.13-0.3$ & $-0.17-0.26$ & $0.11-0.58$ \\
\hline & $\mathrm{p}$ & 0.101 & 0.285 & 0.939 & 0.057 & 0.417 & 0.659 & $0.004 *$ \\
\hline \multirow[t]{3}{*}{ SF } & $\beta$ & 0.237 & 0.240 & -0.014 & -0.134 & 0.128 & -0.074 & 0.173 \\
\hline & $95 \% \mathrm{CI}$ & $0.03-0.44$ & $0.01-0.47$ & $-0.23-0.2$ & $-0.38-0.11$ & $-0.09-0.35$ & $-0.29-0.15$ & $-0.07-0.41$ \\
\hline & $\mathrm{p}$ & $0.025^{*}$ & $0.042 *$ & 0.899 & 0.275 & 0.249 & 0.509 & 0.153 \\
\hline \multirow[t]{3}{*}{ RE } & $\beta$ & 0.224 & 0.155 & 0.193 & -0.088 & 0.070 & -0.068 & 0.242 \\
\hline & $95 \% \mathrm{CI}$ & $0.02-0.43$ & $-0.07-0.38$ & $-0.01-0.4$ & $-0.33-0.15$ & $-0.14-0.28$ & $-0.28-0.15$ & $0.01-0.48$ \\
\hline & $\mathrm{p}$ & $0.034 *$ & 0.177 & 0.067 & 0.462 & 0.520 & 0.535 & $0.043^{*}$ \\
\hline \multirow[t]{3}{*}{$\mathrm{MH}$} & $\beta$ & 0.358 & 0.270 & 0.105 & -0.090 & 0.174 & 0.041 & 0.236 \\
\hline & $95 \% \mathrm{CI}$ & $0.16-0.56$ & $0.06-0.48$ & $-0.09-0.3$ & $-0.32-0.14$ & $-0.03-0.38$ & $-0.16-0.25$ & $0.01-0.46$ \\
\hline & $\mathrm{p}$ & $0.001 *$ & $0.014 *$ & 0.292 & 0.429 & 0.093 & 0.694 & $0.037^{*}$ \\
\hline \multirow[t]{3}{*}{ PCS } & $\beta$ & 0.179 & 0.144 & -0.099 & -0.153 & 0.135 & -0.010 & 0.301 \\
\hline & $95 \% \mathrm{CI}$ & $-0.03-0.39$ & $-0.08-0.37$ & $-0.31-0.11$ & $-0.39-0.09$ & $-0.08-0.35$ & $-0.23-0.21$ & $0.07-0.54$ \\
\hline & $\mathrm{p}$ & 0.092 & 0.211 & 0.345 & 0.205 & 0.214 & 0.925 & $0.012 *$ \\
\hline \multirow[t]{3}{*}{ MCS } & $\beta$ & 0.299 & 0.234 & 0.107 & -0.158 & 0.132 & -0.031 & 0.302 \\
\hline & $95 \% \mathrm{CI}$ & $0.1-0.5$ & $0.02-0.45$ & $-0.09-0.31$ & $-0.39-0.07$ & $-0.08-0.34$ & $-0.24-0.18$ & $0.08-0.53$ \\
\hline & $\mathrm{p}$ & $0.004 *$ & $0.036^{*}$ & 0.292 & 0.176 & 0.210 & 0.768 & $0.010^{*}$ \\
\hline \multirow[t]{3}{*}{ TSF36 } & $\beta$ & 0.267 & 0.211 & 0.016 & -0.169 & 0.145 & -0.024 & 0.328 \\
\hline & $95 \% \mathrm{CI}$ & $0.06-0.47$ & $-0.01-0.43$ & $-0.19-0.22$ & $-0.4-0.06$ & $-0.06-0.35$ & $-0.23-0.19$ & $0.1-0.56$ \\
\hline & $\mathrm{p}$ & $0.011 *$ & 0.060 & 0.872 & 0.149 & 0.170 & 0.823 & $0.005 *$ \\
\hline
\end{tabular}

$\beta$ - standardized beta coefficient; CI - Confidence interval; PF - Physical functioning; RP - Physical roles limitation; BP - Bodily pain; GH - General health; VT - Vitality; SF - Social functioning; RE - Emotional roles limitation; MH - Mental health; PCS - Physical component score; MCS - Mental component score; TSF36 - Total SF-36; ES - Employment status; ED - Education; MS - Marital status; SEC - Socioeconomic status; $\quad * \mathrm{p}<0.05$ 
$\mathrm{p}=0.004)$ and TSF36-Total SF-36 $(\beta=-0.267 ; \mathrm{p}=0.011)$ were better in the ex-POWs group. However, in the adjusted linear regression analysis, captivity remained significant predictor only for the SF-Social functioning $(\beta=-0.240 ; p=0.042)$, MH-Mental health $(\beta=-0.270$; $\mathrm{p}=0.014)$, and MSC-Mental component score $(\beta=-0.234$; $\mathrm{p}=0.036$ ), while the best predictor became socioeconomic status. Low socioeconomic status was a significant predictor of low self-assessed scores for the GH-General health $(\beta=-0.307 ; p=0.010)$, VT-Vitality $(\beta=-0.343 ; p=0.004)$, RE-Emotional roles limitation $(\beta=-0.242 ; p=0.043)$, and $\mathrm{MH}-$ Mental health $(\beta=-0.236$; $\mathrm{p}=0.037$ ), and consequently for the PCS-Physical component score $(\beta=-0.301 ; p=0.012)$, MSC-Mental component score $(\beta=-0.302 ; p=0.010)$ and TSF36-Total SF-36 score $(\beta=-0.328 ; \mathrm{p}=0.005)$.

\section{DISCUSSION}

\section{Sociodemographic Characteristics}

In this study, the Croatian war veterans were divided into two groups, considering whether they were ex-POWs or not (control group). All participants were males, most are now aged between 45 and 64 years, and have a high school level of education. A significant difference was in the marital status. The number of married subjects in the ex-POWs group was significantly higher in relation to the control group which is characterized by a large number of divorced persons. Studies dealing with the functioning of veterans in different domains of life point to the difficult functioning of patients with PTSD. Studies of the interpersonal functioning of PTSD patients point to difficulties in marital and family functioning, or difficulties in emotional relationships (Amaya-Jackson et al. 1999). One of these studies confirms that $70 \%$ of veterans have disturbed partnerships (Riggs et al. 1998) and that veterans with PTSD are more inclined to break partner relationships (Kulka et al. 1990). Besides impaired functioning within interpersonal, marital and emotional relationships, PTSD is also associated with disrupted work functioning. Veterans suffering from PTSD are more likely to lose their jobs or become homeless (Magruder et al. 2004, Smith et al. 2005, O'Connell et al. 2008). In this study, low socioeconomic status was significantly more prevalent in the control group, which can be explained by a larger number of unemployed persons in this group indicating a more regular income of the ex-POWs.

\section{The Intensity of PTSD Symptoms}

PTSD symptoms were more pronounced in the control group. This is contrary to the expectations and results of some other investigations, where more intense PTSD symptoms were detected in the ex-POWs many years after detention. A comparison study of Israeli exPOWs 18 and 30 years after captivity with veterans with only combat experience, points to the more significant intensity of PTSD symptoms in ex-POWs (Solomon et al. 2008). Similar results were found in studies dealing with the consequences of captivity on mental health (Engdahl et al. 1998). In our study, we did not deal with individual traumatic events, and some research suggests that the severity of PTSD is associated with the number of traumatic experiences (Marušić et al. 1995). Many studies show a high rate of morbidity among ex-POWs (e.g. World War II and Vietnam War ex-POWs) compared to veterans who were not detainees and had a combat experience (Nice et al. 1996).

\section{Comorbidity}

PTSD is not only a mental disorder but is associated with an increased risk for physical illness and early mortality (McFarlane 2010). Frequent physical comorbidity with PTSD has been confirmed in many studies in which the results point to more frequent physical illness in PTSD veterans compared to those without PTSD or in comparison with the general population (Boscarino 1997, Otoole \& Cats 2008). The results of our research indicate the highest incidence of musculosceletal, cardiovascular, and gastrointestinal diseases in both subject groups. Regarding somatic comorbidity, endocrine and metabolic diseases were the only group of diseases with a significant difference between groups, and with the higher incidence in the group of ex-POWs. Neurological diseases occurred more frequently in the control group.

Increased risk for cardiovascular diseases associated with PTSD has been confirmed in other studies. Edmonson et al. (2013) have described a high prevalence of myocardial infarction and increased risk of death from cardiovascular diseases in patients with PTSD. Somatic comorbidity research in veterans 15 years after the war also point to frequent comorbidity of PTSD with cardiovascular diseases with other physical diseases such as musculoskeletal, dermatological, pulmonary, gastrointestinal and metabolic disorders (Britvić et al. 2015). A study involving hospitalized Croatian veterans suffering from PTSD describes diabetes, hypertension, ulcerative disease and hyperlipidemia as the most common comorbid physical conditions (Filaković et al. 1997). Jakovljević et al. (2007) confirm the association between PTSD and metabolic disorders. A large US study of comorbidity of anxiety disorders with physical illness indicates high PTSD comorbidities, particularly with neurological, immune, cardiovascular, and bone and joint diseases (Sareen et al. 2005). Frequent comorbidity is also caused by the bad living habits in veterans suffering from PTSD such as excessive smoking, alcohol consumption, inadequate physical activity and excessive body weight (Zen et al. 2012, Bartoli et al. 2015). Studies investigating the physical health of exPOWs show different results, some of which point to a possible manifestation of the consequences for physical health several decades after captivity (Page \& Brass 2001). In addition, some associate detention conditions (treatment of detainees, poor hygiene and nutrition) with long-lasting detrimental effects on the health of ex- 
POWs (Engdahl et al. 1993). A survey involving Australian World War II detainees showed a significant deterioration of physical health, more physical symptoms, and increased usage of drugs. Ex-POWs were more likely to have gastrointestinal, musculoskeletal, and cognitive disorders, and more extrapyramidal signs associated with ataxia (Creasey et al. 1999). A study also conducted on Australian POWs, and deals with the physical health consequences of injuries and torture during captivity, suggests the more frequent occurrence of the chronic peripheral nerve disorders, spinal and joint diseases, and increased prevalence of peptic ulcer disease (Venn \& Guest 1991). The high incidence of the duodenal ulcer at World War II ex-POWs has been observed even 40 years after detention, and is associated with losing body mass and nutritional deficit disorder during captivity (Tennant et al. 1986).

\section{Health-Related Quality of Life (HRQoL)}

In this study, HRQoL was assessed with an SF-36 questionnaire, which is often used in epidemiological studies to evaluate the health and impact of various diseases on the functioning and QoL of a particular population (Higgins \& Campanera 2011). Ex-POWs and the control group were compared through the eight dimensions of health summarized in two profiles: mental and physical health. The results showed that nondetainees had lower scores for mental health profile and overall HRQoL. The influence of PTSD on HRQoL and functionality across different functional areas has been proven often (Holbrook et al. 2001, Rapaport et al. 2005). The HRQoL is the subject of many types of research in the last two decades, and many deal with the QoL of veterans suffering from PTSD (Kučukalić et al. 2007). Lower HRQoL in patients with PTSD is also caused by frequent comorbidities and poor living habits, alcohol consumption, and smoking (Schnurr et al. 2006, Aversa et al. 2012). One of the studies describes lower HRQoL in veterans suffering from PTSD in comorbidity with chronic physical pain compared to those without PTSD or without chronic pain (Braš et al. 2011).

The results of correlation analysis in our study showed a significant correlation between several diagnostic groups of the physical diseases and HRQoL. There was a significant association of deteriorated HRQoL with the cardiovascular, gastrointestinal, neurological and musculosceletal diseases. The cardiovascular diseases affected physical functioning and intensity of bodily pain, while gastrointestinal diseases affected the general health impression, vitality, and social functioning. The intensity of bodily pain and poor perception of the vitality and mental health were also associated with the neurological diseases. Difficult physical functioning was also associated with musculosceletal diseases. In our research, it was not confirmed that ex-POWs had significantly more physical diseases compared with non-detained veterans, which is contrary to the results of some other studies (Hourani \& Hilton 2002).
Non-detainees have shown poorer social functioning and emotional difficulties with the pronounced PTSD symptoms related to intrusion, negative alterations in cognitions and mood, and alterations in arousal and reactivity. Many studies have been focused on the influence of different clusters of PTSD symptoms on functioning and QoL (Taylor et al. 2006). PTSD has been confirmed as a significant predictor of impaired functioning, or deteriorated HRQoL, especially in the mental health component. The results of a Canadian study comparing veterans with PTSD and those without PTSD confirmed the significant association of PTSD and impaired QoL, especially in the dimension that relates to the sense of emotional well-being. In the same study, the QoL in the profile of physical health was not significantly impaired (Richardson et al. 2008). Other studies indicate an association between poor health and HRQoL in both component, physical and mental (Malik et al. 1999, Baret et al.2002).

The results of regression analysis in our study, which examined the relationship between individual sociodemographic characteristics and dimensions of health, clearly pointed to low socioeconomic status as a significant predictor of poor health and HRQoL in its entirety and in individual dimensions. The low socioeconomic status was also related to more intense PTSD symptoms among subjects. Ex-POWs assessed their health as better compared to the control group, which suggests that socioeconomic indicators could be better predictors of HRQoL than exposure to detention trauma. Such results point to the importance of veterans' post-war care and social support. As the HRQoL of exPOWs was better than in non-detained veterans, perhaps more social support was provided to ex-POWs through various projects and support programs after release from captivity. In this paper, we did not deal with the issues of social support, but its relevance is indicated by the results of other studies (Hunt \& Robbins 2001, Klarić et al. 2008, Yazicioğlu et al. 2006). The adequate psychosocial assistance is an important aspect of positive action on the post-traumatic environment and can prevent PTSD symptoms or other traumatic psychiatric disorders (Kozarić-Kovačić et al. 1995).

\section{Limitations of the study}

The study sample size was limited by the number of ex-POWs treated for PTSD in the institution where the survey was conducted. Particular traumatic events, and the duration of detention were not considered. The greatest limitation of the study was the lack of information on possible differences in the types and quality of postwar social support among ex-POWs and non-detained veterans. The availability of this information could be crucial in overcoming the symptoms of PTSD and ensuring an adequate level of QoL. In this paper, we did not deal with psychiatric comorbidity and its influence on the HRQoL. 


\section{CONCLUSIONS}

According to the results of our study, the experience of war captivity did not cause a long-term higher intensity of PTSD symptom, greater physical comorbidity, nor significantly impaired HRQoL compared to nondetained veterans. Low socioeconomic status has proved to be the most significant predictor of poorer QoL, impaired functioning, and physical comorbidity. Since low socioeconomic status was more pronounced in the non-detained veterans, their QoL was also more impaired. PTSD was associated with the HRQoL, whether the veterans were ex-POWs or not. Further research will provide new insight into the predictors of the HRQoL of veterans of the Croatian Homeland War.

\section{Acknowledgements: None.}

Conflict of interest: None to declare.

\section{Contribution of individual authors:}

Melita Jukić: study conception and design, data acquisition, analysis and interpretation of data, drafting manuscript;

Pavo Filaković, Ivan Požgain \& Trpimir Glavina: study conception and design, critical revision.

\section{References}

1. Ahmadi K, Shahidi S, Nejati V, Karami $G$ \& Masoomi M: Effects of chronic illness on the quality of life in psychiatric out patients of the Iraq - Iran War. Iran J Psychiatry 2013; 8:7-13

2. Al-Turkait FA \& Ohaeri JU: Prevalence and correlates of posttraumatic stress disorder among Kuwaiti military men according to level of involvement in the first Gulf War. Depress Anxiety 2008; 25: 932-941.

3. Amaya Jackson L, Davidson JR, Hughes DS, Swartz M, Reynolds V, George LK et al.: Functional impairment and utilization of services associated with post traumatic stress in the community. J Trauma Stress 1999;12:709-24.

4. American Psychiatric Association: Diagnostic and statistical manual of mental disorders: DSM-IV. Fourth Edition. Arlington, VA: American Psychiatric Publishing, 1994

5. American Psychiatric Association: Diagnostic and statistical manual of mental disorders: DSM-5. Fifth Edition. Arlington, VA: American Psychiatric Publishing, 2013

6. Apolone $G \&$ Mosconi P: Review of the concept of quality of life assessment and discussion of the present trend in clinical research. Nephrol Dial Transplant 1998; 13(Suppl 1):65-69

7. Aversa LH, Stoddard JA, Doran NM, Au S, Chow B, McFall $M$ et al.: PTSD and depression as predictors of physical health-related quality of life in tobaccodependent veterans. J Psychosom Res 2012;73:185-90

8. Barrett DH, Doebbeling CC, Schwartz DA, Voelker MD, Falter KH, Woolson RF et al.: Posttraumatic stress disorder and self-reported physical health status among U.S. military personnel serving during the Gulf War period. Psychosomatics 2002; 43:195-205
9. Bartoli F, Crocamo C, Alamia A, Amidani F, Paggi E, Pini E et al.: Posttraumatic stress disorder and risk of obesity: Systematic review and meta-analysis. J Clin Psychiatry 2015; 76:1253-1261

10. Başoğlu M: A multivariate contextual analysis of torture and cruel, inhuman, and degrading treatments: Implications for an evidence based definition of torture. Am J Orthopsychiatry 2009; 79: 135-145.

11. Boscarino JA: Diseases among men 20 years after exposure to severe stress: implications for clinical research and medical care. Psychosom Med 1997; 59:605-614.

12. Braš M, Milunović V, Boban M, Brajković L, Benković $V$, Dordevic $V$ et al.: Quality of life in Croatian Homeland War (1991-1995) veterans who suffer from post-traumatic stress disorder and chronic pain. Health Qual Life Outcomes 2011; 9:56

13. Britvić D, Antičević V, Kaliterna M, Lušić L, Beg A, Brajević-Gizdić I et al: Comorbidities with posttraumatic stress disorder (PTSD) among combat veterans: 15 years postwar analysis. Int J Clin Heal Psychol 2015; 15:81-92

14. Buckley TC, Mozley SL, Bedard MA, Dewulf A-C \& Greif J: Preventive health behaviors, health-risk behaviors, physical morbidity, and health-related role functioning impairment in veterans with post-traumatic stress disorder. Mil Med 2004; 169:536-540

15. Creasey H, Sulway MR, Dent O, Broe GA, Jorm A \& Tennant $C$ : Is experience as a prisoner of war a risk factor for accelerated age- related illness and disability? J Am Geriatr Soc 1999; 47:60-64

16. Edmondson D, Kronish IM, Shaffer JA, Falzon L \& Burg MM: Posttraumatic stress disorder and risk for coronary heart disease: A meta-analytic review. Am Heart J 2013; 166: 806-814

17. Engdahl B, Dikel TN, Eberly $R$ \& Blank A: Comorbidity and course of psychiatric disorders in a community sample of former prisoners of war. Am J Psychiatry 1998; 155:1740-1745

18. Engdahl BE, Harkness AR, Eberly RE, Page WF \& Bielinski J: Structural models of captivity trauma, resilience, and trauma response among former prisoners of war 20 to 40 years after release. Soc Psychiatry Psychiatr Epidemiol 1993; 28:109-115

19. Filakovic P, Barkic J, Kadoic D, Crncevic Orlic Z, Grguric Radanovic L, Karner I et al.: Biological parameters of posttraumatic stress disorder. Psychiatr Danub 1997; 9:207-211

20. Herman JL: Complex PTSD: A syndrome in survivors of prolonged and repeated trauma. J Trauma Stress 1992; 5:377-391

21. Higgins $P \&$ Campanera JM: (Sustainable) quality of life in English city locations. Cities 2011; 28:290-299

22. Holbrook TL, Hoyt DB, Stein MB \& Sieber WJ: Perceived threat to life predicts posttraumatic stress disorder after major trauma: risk factors and functional outcome. $J$ Trauma Inj Infect Crit Care 2001; 51:287-293

23. Hourani LL \& Hilton $S$. The long-term psychiatric sequelae of the prisoner of war experience: Findings from Operation Homecoming Vietnam Veterans. Naval Health Research Center; San Diego, CA, 2002; Report No. $02-18$

24. Hunt $N$ \& Robbins I: World War II veterans, social support, and veterans' associations. Aging Ment Heal 2001; 5:175-182 
25. Jakovljević M, Crnčević Ž, Ljubičić D, Babić D, Topić $R$ \& Šarić M: Mental disorders and metabolic syndrome: A fatamorgana or warning reality? Psychiatr Danub 2007; 19:76-86

26. Klarić M, Frančišković T, Klarić B, Krešić M, Grković J, Lisica ID et al.: Social support and PTSD symptoms in war-traumatized women in Bosnia and Herzegovina. Psychiatr Danub 2008; 20:466-473

27. Kozarić-Kovačić, D, Folnegović-Šmalc, V, Marušić, A \& Arcel TL: Experiences after two years of providing psycho-social help to women-victims of war and their families from Bosnia and Herzegovina and Croatia. Croat Med J 1995; 6:67-69

28. Kučukalić A, Bravo-Mehmedbašić A, Fadilpašić $S \&$ Džubur-Kulenović A: War trauma and their impact on the quality of life. Med. Arh 2007; 61:1-80:26

29. Kulka R, Schlenger W, Fairbank J, Hough R, Jordan B, Marmar $C$ et al.: Trauma and the Vietnam War generation: Report of findings from the National Vietnam Veterans Readjustment Study. Brunner/Mazel, Philadelphia, 1990

30. Magruder KM, Frueh BC, Knapp RG, Johnson MR, Vaughan JA, Carson TC, et al.: PTSD symptoms, demographic characteristics, and functional status among veterans treated in VA primary care clinics. J Trauma Stress 2004; 17:293-301

31. Malik ML, Connor KM, Sutherland SM, Smith RD, Davison RM \& Davidson JRT: Quality of life and posttraumatic stress disorder: A pilot study assessing changes in SF-36 scores before and after treatment in a placebo-controlled trial of fluoxetine. $J$ Trauma Stress 1999; 12:387-393

32. Marušić A, Kozarić-Kovačić D, Folnegović-Šmalc $V$, Ljubin T. \& Ljubin S.: Use of two PTSD scales in assessing PTSD refugees and displaced persons from Bosnia and Herzegovina and Croatia, Psychologische Beitraege, 1995; 37: 232-235

33. McFarlane AC: The long-term costs of traumatic stress: Intertwined physical and psychological consequences. World Psychiatry 2010; 9:3-10

34. Mittal D, Fortney JC, Pyne JM, Edlund MJ \& Wetherell $J L$ : Impact of comorbid anxiety disorders on healthrelated quality of life among patients with major depressive disorder. Psychiatr Serv 2006; 57:1731-1737

35. Neria Y, Solomon Z \& Dekel R: An eighteen-year followup study of Israeli prisoners of war and combat veterans. J Nerv Ment Dis 1998; 186:174-182

36. Nice DS, Garland CF, Hilton SM, Baggett JC \& Mitchell RE. Long-term health outcomes and medical effects of torture among US navy prisoners of war in Vietnam. J Am Med Assoc 1996; 276:375-381

37. O'Connell MJ, Kasprow $W$ \& Rosenheck RA: Rates and risk factors for homelessness after successful housing in a sample of formerly homeless veterans. Psychiatr Serv 2008; 59:268-275.

38. O'Toole BI \& Catts SV: Trauma, PTSD, and physical health: an epidemiological study of Australian Vietnam veterans. J Psychosom Res 2008; 64:33-40

39. Page WF \& Brass LM: Long-term heart disease and stroke mortality among former American prisoners of war of World War II and the Korean Conflict: results of a 50year follow-up. Mil Med 2001; 166:803-808

40. Rapaport MH, Clary C, Fayyad R \& Endicott J: Qualityof-life impairment in depressive and anxiety disorders. Am J Psychiatry 2005; 162:1171-1178
41. Rapaport MH, Endicott J \& Clary CM: Posttraumatic stress disorder and quality of life: Results across 64 weeks of sertraline treatment. J Clin Psychiatry 2002; 63:59-65

42. Richardson JD, Long ME, Pedlar D \& Elhai JD: Posttraumatic stress disorder and health-related quality of life among a sample of treatment- and pension-seeking deployed Canadian forces peacekeeping veterans. Can J Psychiatry 2008; 53:594-600

43. Riggs DS, Byrne CA, Weathers FW \& Litz BT: The quality of the intimate relationships of male Vietnam veterans: Problems associated with posttraumatic stress disorder. $J$ Trauma Stress 1998; 11:87-101

44. Rintamaki LS, Weaver FM, Elbaum PL, Klama EN \& Miskevics SA: Persistence of traumatic memories in World War II prisoners of war. J Am Geriatr Soc 2009; 57:2257-2262

45. Sareen J, Cox BJ, Clara I \& Asmundson GJG: The relationship between anxiety disorders and physical disorders in the U.S. National Comorbidity Survey. Depress Anxiety 2005; 21:193-202

46. Schnurr PP, Hayes AF, Lunney CA, McFall M\& Uddo M: Longitudinal analysis of the relationship between symptoms and quality of life in veterans treated for posttraumatic stress disorder. J Consult Clin Psychol 2006; 74:707-713

47. Schnurr PP, Lunney CA, Bovin MJ \& Marx BP: Posttraumatic stress disorder and quality of life: Extension of findings to veterans of the wars in Iraq and Afghanistan. Clin psychol Rev 2009; 29:727-735.

48. Smith MW, Schnurr PP \& Rosenheck RA: Employment outcomes and PTSD symptom severity. Ment Health Serv Res 2005; 7:89-101

49. Solomon Z, Dekel R \& Mikulincer M: Complex trauma of war captivity: A prospective study of attachment and post-traumatic stress disorder. Psychol Med 2008; 38:1427-1434

50. Solomon Z, Greene T, Ein-Dor T, Zerach G, Benyamini $Y$ $\&$ Ohry A: The long-term implications of war captivity for mortality and health. J Behav Med 2013; 37:849-859

51. Solomon Z, Horesh D, Ein-Dor T \& Ohry A: Predictors of PTSD trajectories following captivity: A 35-year longitudinal study. Psychiatry Res 2012; 199:188-194

52. Swan A, Watson HJ \& Nathan PR: Quality of life in depression: An important outcome measure in an outpatient cognitive-behavioural therapy group programme? Clin Psychol Psychother 2009; 16:485-496

53. Taylor S, Wald J \& Asmundson GJG: Factors Associated with Occupational Impairment in People Seeking Treatment for Posttraumatic Stress Disorder. Can J Community Ment Heal 2006; 25:289-301.

54. Tennant CC, Goulston KJ \& Dent OF: The psychological effects of being a prisoner of war: Forty years after release. Am J Psychiatry 1986; 143:618-621

55. The Government of the Republic of Croatia: National program of psychosocial help for the victims in the Homeland War [in Croatian]. Zagreb: Ministry of Croatian Veterans, 1999

56. The Government of the Republic of Croatia: National program of psychosocial help for the participants and victims of the Homeland War, World War II, and returnees from peacekeeping missions [in Croatian]. Zagreb: Ministry of Croatian Veterans, 2014

57. Venn AJ \& Guest CS: Chronic morbidity of former prisoners of war and other Australian veterans. Med J Aust 1991; 155:705-712 
58. Ware JE, Kosinski $M$ \& Dewey JE. How to Score Version2 of the SF-36® Health Survey (Standard \& Acute Forms). Second Edition. Lincoln, QualityMetric Incorporated, 2001

59. Ware JE, Kosinski $M \&$ Gandek B: SF-36® Health Survey Manual \& Interpretation Guide. Boston, The Health Institute, 1993

60. Weathers FW, Marx BP, Friedman MJ \& Schnurr PP: Posttraumatic stress disorder in DSM-5: New criteria, new measures, and implications for assessment. Psychol Inj Law 2014; 7:93-107

61. Weiss MG, Saraceno B, Shekhar $S$ \& Ommeren M: Mental health in the aftermath of disasters: Consensus and controversy. J Nerv Ment Dis 2003; 191:611-615

62. WHOQOL Group: The World Health Organization Quality of Life Assessment (WHOQOL): development and general psychometric properties. Soc Sci Med 1998; 46:1569-1585

63. World Health Organization: The ICD-10 Classification of Mental and Behavioural Disorders: Clinical Descriptions and Diagnostic Guidelines. Geneva, World Health Organization, 1992

64. World Health Organization-Division of Mental Health and Prevention of Substance Abuse: WHOQOL: measuring quality of life. Geneva, World Health Organization, 1997

65. Wortmann JH, Jordan AH, Weathers FW, Resick PA, Dondanville KA, Hall-Clark et al.: Psychometric analysis of the PTSD Checklist-5 (PCL-5) among treatmentseeking military service members. Psychol Assess 2016; 28:1392-1403

66. Yazicioğlu K, Duyan V, Karataş K, Özgül CA, Yilmaz MB, Duyan GÇ et al.: Effects of sociodemographic characteristics, illness process, and social support on the levels of perceived quality of life in veterans. Mil Med 2006; 171:1083-1088

67. Zen AL, Whooley MA, Zhao S\& Cohen BE: Post-traumatic stress disorder is associated with poor health behaviors: findings from the heart and soul study. Health Psychol 2012; 31: 194-201

Correspondence:

Melita Jukić, MD

Head of the Department of Psychiatry of the County General Hospital Vukovar and Croatian Veterans' Hospital Županijska ulica 35, HR-32000, Vukovar, Croatia E-mail:mjuki17@gmail.com 